\title{
Acoustic astrometry with a VLBI-like interferometer
}

\author{
I. Martí-Vidal and J. M. Marcaide \\ Dpt. Astronomia i Astrofísica, Universitat de València, C/ Dr. Moliner 50, 46100 Burjassot, \\ Valencia (SPAIN) \\ email: i.marti-vidal@uv.es
}

\begin{abstract}
We show how loud-speakers, home digital recorders, and a common personal computer can be used to emulate VLBI observations on a small scale. These audio-VLBI observations allow for single-field astrometry (sources within the same interferometric field), differential group-delay astrometry, etc. These experiments can be set up very easily and in many possible configurations. Students may find these experiments very useful to learn about the innermost details of the interferometric technique.
\end{abstract}

Keywords. techniques: interferometric

\section{Experimental setup}

We performed VLBI-like observations (see, e.g., Thomson, Moran \& Swenson 1986, and references therein, for details on the VLBI technique) using, as signal, sound waves generated by two stereo loud-speakers separated by a distance $d$ (upper side of Fig. 1 (a)). We used home digital audio recorders, located at a distance $D$ from the speakers, as receiving antennas (lower side of Fig. 1 (a)). Our interferometer consisted of 10 antennas, ordered by us in the East-West direction at random distances.

The recorded audio signals were treated following all the usual steps of real VLBI data: down-conversion from the observing frequency using a local oscillator, Nyquist sampling, amplitude digitization, phase rotation, correlation (we performed both, XF and FX correlations), Van Vleck correction, amplitude calibration, and Global Fringe Fitting. All these steps were performed using the software Mathematica (Wolfram 2003).

\section{Single-field astrometry}

When the signal bandwidth is narrow enough (in our case, $10 \mathrm{~Hz}$ with the central frequency at $3 \mathrm{KHz})$, for an appropriate geometry $(d=0.5 \mathrm{~m}$ and $D=2 \mathrm{~m})$ the signals from both speakers will fall within the same fringe. Thus, the resulting visibilities will correspond to the Fourier transform of a double source. In Fig. 1 (b) we show the fringe, in delay space, resulting from the correlation of the data corresponding to one of our shortest baselines. The plot of correlated amplitudes vs. baseline length (Fig. 1 (c)) shows a clear modulation produced by the double source.

Due to the relatively high central frequency and to our low visual precision for the alignment of the signals in delay space, we need to fringe-fit the visibility phases prior to Fourier inverting into the "sky" plane. As shown in Fig. 1 (d), after performing Global Fringe-Fitting and one Hybrid Mapping iteration to the data, we recover a "sky" map with a clear double source (notice that we have also synthesized resolution in the NorthSouth direction, just reproducing the same baselines at different vertical coordinates). 


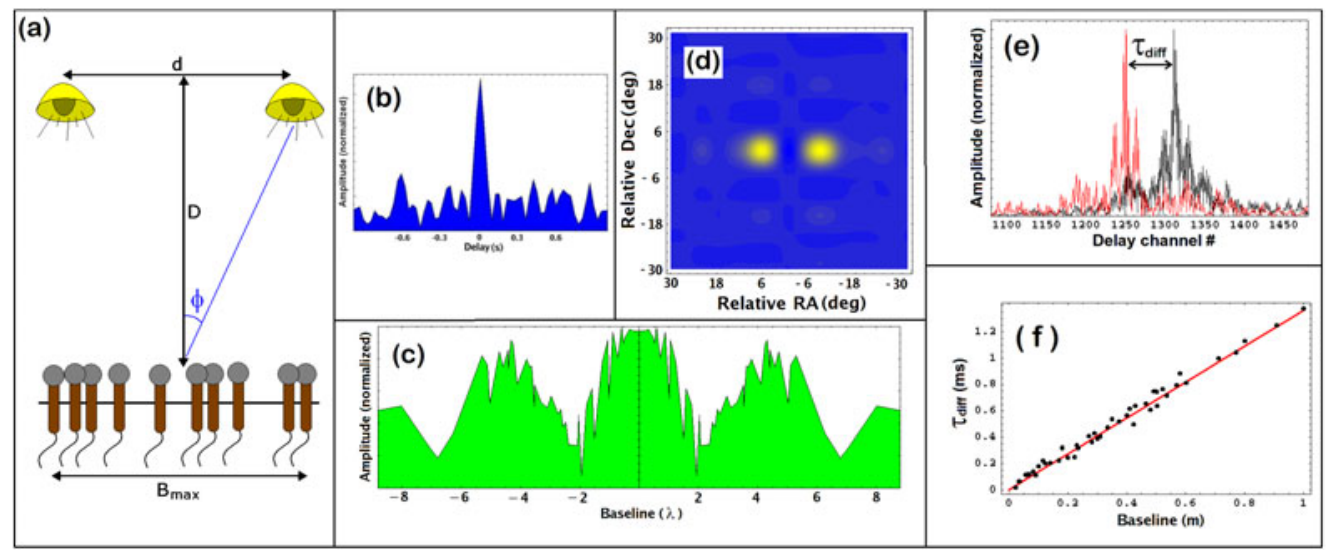

Figure 1. Several figures related to the analysis of our audio-VLBI data (see text).

\section{Group-delay astrometry}

For a wide signal bandwidth $(22 \mathrm{KHz}$ centered at $11 \mathrm{KHz}$ in our case), the signal from each speaker generates its own fringe (i.e., the interferometer sees two single sources instead of one double source), provided measurements are taken for an appropriate geometry $(d=6.5 \mathrm{~m}$ and $D=12 \mathrm{~m})$ with $D$ large enough to grant a fraction of a phase cycle precision in the approximation $D \rightarrow \infty$. In that case, we can separately perform Global Fringe Fitting to the signals coming from each speaker and compute, for each baseline, the differential (group) delays. The latter are the time separations between the fringe peaks generated by both signals (see Fig. 1 (e)).

Figure 1 (e) shows example fringes, in delay space, corresponding to our longest baseline. Fig. 1 (f) shows the results of representing the measured differential group-delays $\left(\tau_{\text {diff }}\right)$ as a function of baseline length for all baselines of our interferometer. The line corresponds to the delay model $\tau_{\text {diff }}=2(B / c) \sin (\phi)$, where $c$ is the speed of sound.

\section{Conclusions}

We have shown that it is possible to perform realistic VLBI emulations using sound waves as signal, home digital recorders as antennas, and a common personal computer as correlator. The observations can be carried out under a large number of different configurations: using the same or different clocks for the recorders, changing the sound pitch, adding spectral lines (i.e., tones) to the continuum, moving the sources during the data acquisition, etc. The educational and training capabilities of these relatively simple hands-on experiments is very promising.

\section{Acknowledgements}

I. M. V. thanks Sergio Jiménez and Diana Dunca for their help setting up these experiments, Loli González and Edén Sorolla for their logistic support, and grant AYA200614986-C02 of the Spanish DGCYT for financial support.

\section{References}

Thomson, A. R., Moran, J. M., \& Swenson, G. W. 1986, Interferometry and Synthesis in Radio Astronomy, Wiley, New York.

Wolfram, S., The Mathematica Book, 2003, 5th Ed., Wolfram Media 